\title{
Dark Energy Constraints on Red-Shift-Based $f(R)$ Gravity
}

\author{
Setareh Dabbaghchian and Reza Saffari \\ Department of Physics, University of Guilan, P.O. Box 41335-1914, Rasht, Iran \\ Correspondence should be addressed to Reza Saffari; rsk@guilan.ac.ir
}

Received 6 December 2012; Accepted 13 January 2013

Academic Editors: D. Kieda and H. Zhao

Copyright (C) 2013 S. Dabbaghchian and R. Saffari. This is an open access article distributed under the Creative Commons Attribution License, which permits unrestricted use, distribution, and reproduction in any medium, provided the original work is properly cited.

\begin{abstract}
We have studied cosmological dynamics in $f(R)$ gravity theory via cosmographic parameters. We have changed variables of field equations from time to red-shift and solved the achieved differential equation analytically for $f(R)$. Then we have used Taylor expansion to find general form of $f(R)$ function around the present day value of scalar curvature. By introducing $F(z)=d f(R) / d R$ we would simplify our calculations; if we consider $F(z)$ as a given function we would restrict our answers of $f(R)$. In this paper we offer a linear form of $F(z)=1+\alpha z$ which leads us to a specific $f(R)$ function, where $\alpha$ is a constant which depends on the present day value of deceleration parameter. As an example, using Taylor expansion coefficients, we have compared our analytically calculated function with reconstructed $f(R)$ function for Dark Energy models. To reconstruct $f(R)$ action for Dark Energy models, we have used corresponding $H(z)$ of each Dark Energy model for calculating Taylor expansion coefficients. As our $F(z)$ function is linear, the Taylor expansion coefficients would be a function of present day value of deceleration parameter.
\end{abstract}

\section{Introduction}

Recent data indicates that the Universe is in an accelerating expansion phase [1-8]. Being responsible for this expansion, several approaches such as positive cosmological constant, Dark Energy, and modified gravity have been introduced. The positive cosmological constant would be defined either geometrically as modifying the left hand side of Einstein equations or as a kinematic term on the right hand side with the equation of state parameter $\omega=-1$; however, the fine tuning problem causes some difficulties [9-16]. There are various scalar field models of Dark Energy [16-33]. These models indeed are the outcomes of modifying the right hand side of the Einstein equations, $G_{\mu \nu}=\chi T_{\mu \nu}$, by considering a source term with an equation of state parameter $\omega<-1 / 3$ which is recognized as Dark Energy. In modified gravity, the right hand side is left unchanged and we modify the left hand side of the Einstein field equations, so we have a large class of theories of gravity [34-42]. Particularly, we are interested in fourth order theories [43-55] based on replacing the scalar curvature $R$ in the Hilbert-Einstein action with a generic analytic function $f(R)$ which should be reconstructed starting from data and physically motivated issues. These models fit both the cosmological data and Solar System constraints in several physically interesting cases [56-65]. Vacuum solutions of $f(R)$ gravity theories is one of interesting subjects which are obtained for constant Ricci scalar [6672], allowing derivation of non constant curvature scalar vacuum solutions. In this paper, following our other work [73], we have considered vacuum solutions of $f(R)$ gravity in a different way which does not assume constant scalar curvature to obtain vacuum solutions.

Vacuum solutions of modified $f(R)$ gravity explain the late time phase transition of cosmological parameters like deceleration parameter just by pure geometry without the need for dark energy companion of the Universe. The pioneering works on reconstruction of modified action through inverse method are done in [66-72]. They developed a general scheme for cosmological reconstruction of modified $f(R)$ gravity in terms of red-shift without using an auxiliary scalar in intermediate calculations. Using this method, it is possible to construct the specific modified gravity which contains any requested FRW cosmology. A number of $f(R)$ gravity examples are constructed where the following background evolutions may be realized: LCDM epoch, deceleration with subsequent transition to effective phantom 
super-acceleration leading to Big Rip singularity, deceleration with transition to transit phantom phase without future singularity, and oscillating Universe. It is important that all these cosmologies may be realized only by modified gravity without the use of any dark components. Then we have tried to reconstruct an appropriate action for the modified gravity through the semi-inverse solution method. We do not assume any FRW cosmology to reconstruct its related $f(R)$ action [63-81]. Indeed, as curvature scalar itself is a function of red-shift, by introducing a linear correction which is also a function of $z$, we can reconstruct Taylor expansion of $f(R)$ action around present time value of red-shift $(z=0)$. For restricting Taylor expansion, we would study the variation of its coefficients from GR.

Capozziello et al. has also studied Taylor expansion of $f(R)$ function in a model independent way where he has shown how it is possible to relate the cosmographic parameters (i.e., the deceleration $q_{0}$, the jerk $j_{0}$, the snap $s_{0}$, and the lerk $l_{0}$ parameters) to the present-day values of $f(R)$ and its derivatives $f^{(n)}(R)=d^{n} f / d R^{n}$ (with $n=1,2,3$ ), thus offering a new tool to constrain such higher order models. Their analysis thus offers the possibility to relate the model independent results coming from cosmography to the theoretically motivated assumptions of $f(R)$ cosmology [75]. But here we have added a new condition to the question which has limited our answers to a certain form of the $f(R)$ function.

In Section 2, we have a briefer review of modified field equations and introduce our $F(z)$ model as a linear function of Red-Shift; we have also tried to reconstruct the $f(R)$ function model independently. In Section 3, we calculate the Taylor expansion of $f(R)$ function via $F(z)$. In Section 4 , we have discussed influence of Taylor expansion coefficients in compare with the first term of expansion. In Section 5, we have reconstructed Taylor expansion for several Dark Energy models and compared the result with our $f(R)$ function which has been calculated in Section 3.

\section{Modified Field Equations}

The action of modified theory of gravity is given by

$$
S=\int d^{4} x \sqrt{-g}\left[f(R)+L_{m}\right]
$$

where $L_{m}$ is the matter action such as radiation, baryonic matter, dark matter, and so on which we do not consider in field equation. In this essay, we consider the flat FriedmannRobertson-Walker, (FRW) background as

$$
d s^{2}=-c^{2} d t^{2}+a(t)^{2}\left[d r^{2}+r^{2} d \theta^{2}+r^{2} \operatorname{Sin}^{2} \theta d \varphi^{2}\right],
$$

so that the gravitational field equations for $f(R)$ gravity are provided by the following form:

$$
\begin{gathered}
-3 \frac{\ddot{a}}{a} f^{\prime}+3 \frac{\dot{a}}{a} \dot{R} f^{\prime \prime}+\frac{1}{2} f=0, \\
{\left[\frac{\ddot{a}}{a}+2\left(\frac{\dot{a}}{a}\right)^{2}\right] f^{\prime}-2 \frac{\dot{a}}{a} \dot{R} f^{\prime \prime}-\dot{R}^{2} f^{\prime \prime \prime}-\ddot{R} f^{\prime \prime}-\frac{1}{2} f=0,}
\end{gathered}
$$

where the over-dot denotes a derivative with respect to $t$, and the prime denotes a derivative with respect to $R, a(t)$ is the scale factor, and $H=\dot{a} / a$ is the Hubble parameter. Eliminating $f$ between (3) and (4) results

$$
-2\left[\frac{\ddot{a}}{a}-\left(\frac{\dot{a}}{a}\right)^{2}\right] f^{\prime}+\frac{\dot{a}}{a} \dot{R} f^{\prime \prime}-\ddot{R} f^{\prime \prime}-\dot{R}^{2} f^{\prime \prime \prime}=0 .
$$

This can be changed in the form of

$$
\ddot{F}-H \dot{F}+2 \dot{H} F=0,
$$

where $F=d f / d R$. Equation (6) is a second order differential equation of $F$ with respect to time, in which both of $F$ and $H$ are undefined. The usual method to solve (6) is based on definition of $f(R)$. Changing the variable of the above equation from $t$ to a new variable like $N$, the number of e-folding, was done in [70-72]. The variable $N$ is related to the red-shift $z$ by $e^{-N}=1+z$. The cosmological dynamic equation is solved by definition of Hubble parameter as a function of $N$ in a general form, then the equation is rewritten by redefinition of the variable from the number of e-folding to the Ricci scalar and solved with respect to the Ricci scalar. Thus they demonstrated that modified $f(R)$ gravity may describe the LCDM epoch without any need for introducing the effective cosmological constant, non-phantom matter, or phantom matter. In this work we would like to replace the variable of (6) by red-shift, $z$, directly to find the $f(R)$ function around $R_{0}$ via Taylor expansion. Each red-shift $z$ has an associated cosmic time $t$ (the time-when objects are observed with redshift $z$ emitting light), so we can replace all the differentials with respect to $t$ by $z$ via

$$
\frac{d}{d t}=\frac{d a}{d t} \frac{d z}{d a} \frac{d}{d z}=-(1+z) H(z) \frac{d}{d z},
$$

where we use $1+z=a_{0} / a$, and we consider $a_{0}=1$, in the present time. Now, we can replace the variable of (6) from $t$ to $z$ by using (7) and obtain a first order differential equation for $H^{2}$ with respect to $z$ as

$$
\frac{d}{d z} H(z)^{2}=P(z) H(z)^{2},
$$

where $P(z)$ depends on the definition of $F(z)$ as

$$
P(z)=\frac{2(1+z)\left(d^{2} F / d z^{2}\right)+4(d F / d z)}{2 F-(1+z)(d F / d z)} .
$$

Now we may solve Hubble parameter that depends on the definition of $F(z)[63,64,73]$. In this paper we consider $F(z)$ as a linear function of $z$ :

$$
F(z)=1+\alpha z
$$

To avoid the Dolgov-Kawasaki instability [82], the second order derivative of $f(R)$ action with respect to Ricci scalar should be positive for different values of $\alpha$. Solving (8), using linear correction equation (10), the Hubble parameter has been found as

$$
H(z)^{2}=H_{\alpha}^{2}\left[1-\frac{\alpha}{2}(1+z)\right]^{4}
$$


in which $H_{\alpha}^{2}=H_{0}^{2} /(1-\alpha / 2)^{4}$. Using Ricci scalar definition, $R=6\left(\dot{H}+2 H^{2}\right)$, the scalar curvature would be

$$
R(z)=R_{\alpha}\left[1-\frac{\alpha}{2}(1-z)\right]^{3}
$$

in which

$$
R_{\alpha}=\frac{12(1-\alpha) H_{0}^{2}}{(1-\alpha / 2)^{4}} .
$$

Eliminating $z$ between (10) and (12) leads us to reach

$$
F(R)=2\left(\frac{R}{R_{\alpha}}\right)^{1 / 3}+\alpha-1 .
$$

In the case of $\alpha=0$ or $R_{\alpha}=R_{0}$, we would like to recover de'Sitter solution. Using (12) we obtain $R=R_{0}$ and then $F(R)=1$ which is equal to the GR or de'Sitter model. Calculating $f(R)$ by integrating of (14) with respect to $R$, we reach

$$
f(R)=R\left(\frac{3}{2}\left(\frac{R}{R_{\alpha}}\right)^{1 / 3}+\alpha-1\right)+C,
$$

where $C$ is the constant of integration. Since in the absence of correction term, $\alpha$, we should recover de'Sitter model $\left(f_{d}(R)=R-2 \Lambda\right)$, we compare the value of de'Sitter action with the action of (15) when $\alpha=0$ or $R=R_{0}$. Since the scalar curvature of de'Sitter Universe is a constant, then we can determine the value of $f_{d}(R)$ in the presence of constant curvature. If we solve the standard algebraic equation of $f(R)$ gravity as $2 f_{d}(R)-R F_{d}(R)=0$ we can find $R_{0}=4 \Lambda$. Then the value of de'Sitter action in the case of $R=R_{0}$ is $f_{d}\left(R_{0}=\right.$ $4 \Lambda)=2 \Lambda$ which is equal to $R_{0} / 2$. For the case of our obtained action from (15), we have $f\left(R=R_{0}\right)=R_{0} / 2+C$ when $\alpha=0$. Since we would like to have the same solution for de'Sitter model and our obtained action in present of $R=R_{0}$ we force constant of integration, $C$, to be equal to zero [73]. One of main late time cosmological constraint which is tested for redshift-based correction of $f(R)$ gravity is SNeIa distance module with respect to redshift constraint. The result of this comparison is shown in Figure 3 [73].

\section{Taylor Expansion of $f(R)$ via General Function of $F(z)$}

As we are interested in finding appropriate action without solving field equation, here we have used Taylor expansion of $f(R)$ function around $R=R_{0}=R(z=0)$, However, as we are using this method, our reconstructed $f(R)$ is acceptable just around present day value of curvature scalar:

$$
\begin{aligned}
f(R)= & f\left(R_{0}\right)+\left.\left(R-R_{0}\right) \frac{d f}{d R}\right|_{R_{0}}+\left.\frac{\left(R-R_{0}\right)^{2}}{2 !} \frac{d^{2} f}{d R^{2}}\right|_{R_{0}} \\
& +\left.\frac{\left(R-R_{0}\right)^{3}}{3 !} \frac{d^{3} f}{d R^{3}}\right|_{R_{0}}+\cdots
\end{aligned}
$$

and as $R=R(z)$, coefficients of Taylor expansion to the third order would be find as

$$
\begin{gathered}
\frac{d}{d R} f(R)=F(z), \\
\frac{d^{2}}{d R^{2}} f(R)=\left(\frac{d R}{d z}\right)^{-1} \frac{d F}{d z} \\
\frac{d^{3}}{d R^{3}} f(R)=\left(\frac{d R}{d z}\right)^{-2} \frac{d^{2} F}{d z^{2}}-\frac{d^{2} R / d z^{2}}{(d R / d z)^{3}} \frac{d F}{d z} .
\end{gathered}
$$

Via (7), (8), and definition of Ricci scalar, $R=6\left(\dot{H}+2 H^{2}\right)$, we can rewrite $R$ as a function of $z$ :

$$
R(z)=3 H^{2}[4-(1+z) P(z)]
$$

We can normalize $R(z)$ to $H_{0}$ :

$$
\begin{aligned}
R(z) & =\frac{3 H(z)^{2}}{H_{0}^{2}}[4-(1+z) P(z)] \\
& =3 h(z)^{2}[4-(1+z) P(z)] .
\end{aligned}
$$

Finding the first term of Taylor expansion, $f\left(R_{0}\right)$ we use (3) so it also should be written with respect to $z$ :

$$
f(R(z))=6 h(z)^{2}\left[q(z) F(z)+(1+z) \frac{d F(z)}{d z}\right],
$$

where $q(z)=-1-\left(\dot{H} / H^{2}\right)$ is the deceleration parameter that after replacing all the differentials with respect to $t$ by $z$ via (7) would be as

$$
q(z)=-1+\frac{1+z}{2 H^{2}} \frac{d H^{2}}{d z}
$$

or

$$
q(z)=-1+\frac{1+z}{2} P(z)
$$

Finally the constant term of Taylor expansion would be obtained by substituting $z=0$ in (20). Using the linear modification $F(z)=1+\alpha z, R$ derivatives for this function in $z=0$ would be as

$$
\begin{aligned}
& R_{0}=\frac{24(1-\alpha) H_{0}^{2}}{(2-\alpha)}, \\
& R^{\prime}(z=0)=\frac{72(1-\alpha) \alpha H_{0}^{2}}{(2-\alpha)^{2}}, \\
& R^{\prime \prime}(z=0)=\frac{144(1-\alpha) \alpha^{2} H_{0}^{2}}{(2-\alpha)^{3}} .
\end{aligned}
$$




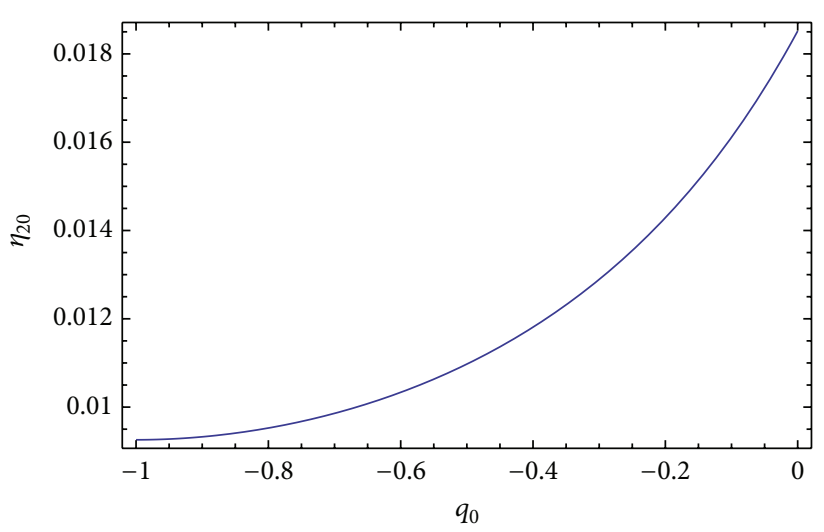

(a)

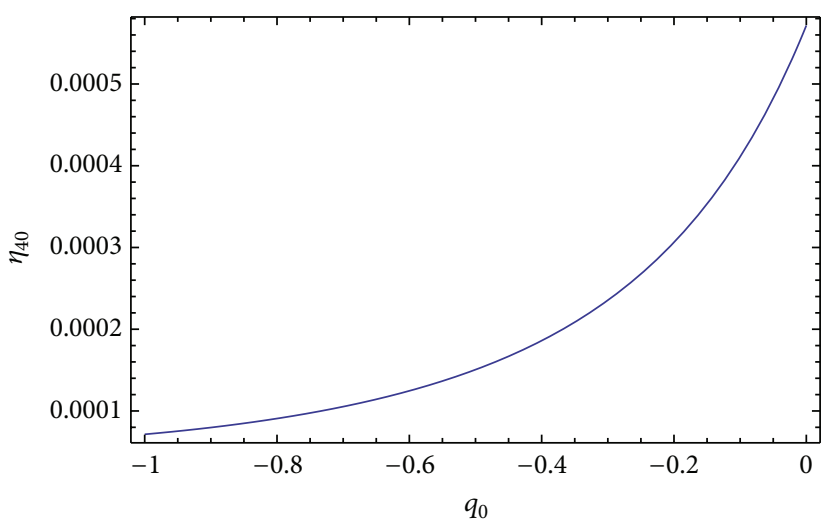

(c)

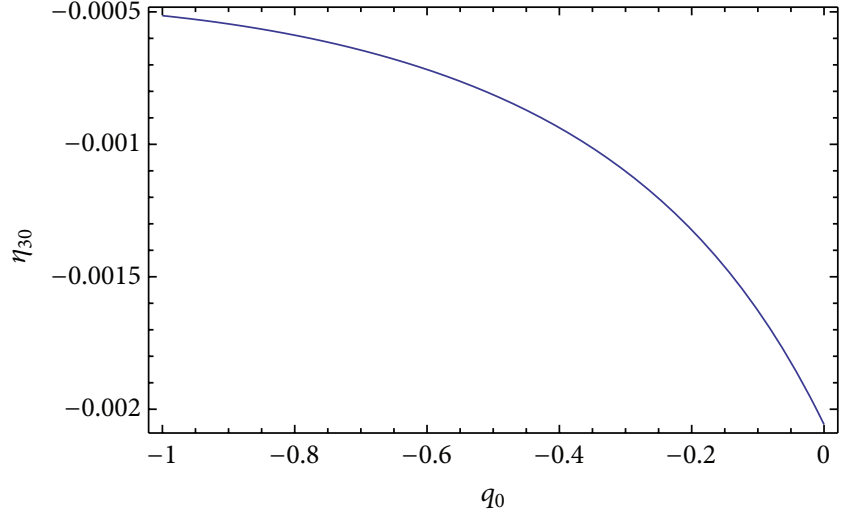

(b)

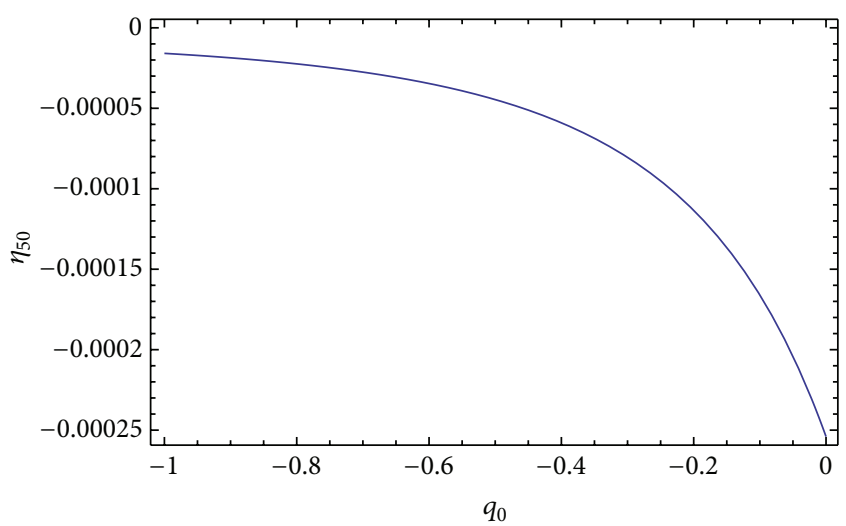

(d)

FIGURE 1: The dimensionless ratio between the present-day values of $f^{(i)}(R)$ and $f(R)$ as a function of $q_{0}$.

Hence, using (16) Taylor expansion coefficients would be

$$
\begin{gathered}
f\left(R_{0}\right)=-\frac{6\left(\alpha^{2}+\alpha-2\right) H_{0}^{2}}{2-\alpha}, \\
\left.\frac{d}{d R} f(R)\right|_{R_{0}}=1, \\
\left.\frac{d^{2}}{d R^{2}} f(R)\right|_{R_{0}}=\frac{(2-\alpha)^{2}}{72(1-\alpha) H_{0}^{2}}, \\
\left.\frac{d^{3}}{d R^{3}} f(R)\right|_{R_{0}}=-\frac{(2-\alpha)^{3}}{2592(1-\alpha)^{2} H_{0}^{4}} .
\end{gathered}
$$

\section{Order of Taylor Expansion}

In this section we keep Taylor expansion of $f(R)$ action up to the third order term and we ignore higher order modifications. Therefore, $f(R)$ Taylor expansion coefficients have been studied as a function with respect to present day value of deceleration parameter. For linear correction, $F(z)=1+\alpha z$, $q(z)$ would be obtained by $(22)$ as

$$
q(z=0)=-1+\frac{2 \alpha}{2-\alpha} .
$$

Therefore

$$
\alpha=\frac{2\left(1+q_{0}\right)}{3+q_{0}} .
$$

Rewriting $f(R)$ Taylor expansion coefficients with respect to $q_{0}$, via $(26)$ and then introducing function $\eta_{i 0}\left(q_{0}\right)=$ $h_{0}^{2 i} f^{(i)}\left(q_{0}\right) / f\left(q_{0}\right)$ [75], in which $i=2,3,4,5, \ldots$ is the order of differentiation, we have:

$$
\begin{aligned}
& \eta_{20}\left(q_{0}\right)=\frac{1}{27\left(2-3 q_{0}+q_{0}^{3}\right)}, \\
& \eta_{30}\left(q_{0}\right)=\frac{1}{243\left(-1+q_{0}\right)^{3}\left(2+q_{0}\right)}, \\
& \eta_{40}\left(q_{0}\right)=\frac{5}{4374\left(-1+q_{0}\right)^{4}\left(2+q_{0}\right)}, \\
& \eta_{50}\left(q_{0}\right)=\frac{10}{19683\left(-1+q_{0}\right)^{5}\left(2+q_{0}\right)} .
\end{aligned}
$$




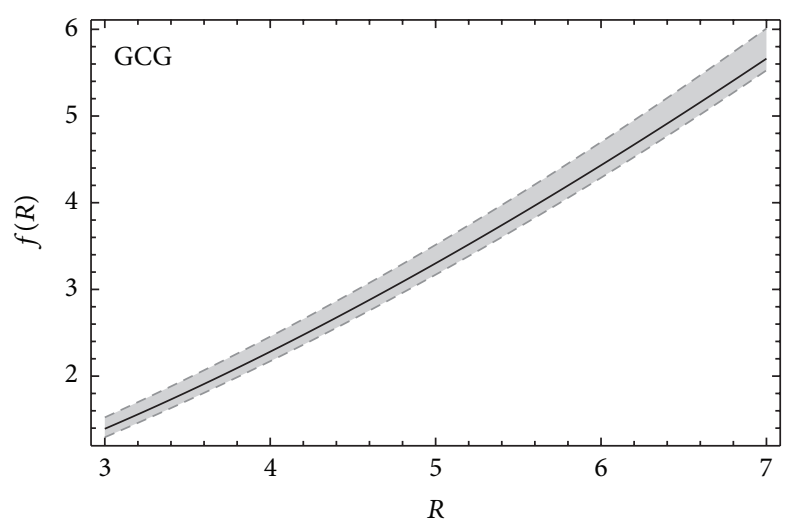

(a)

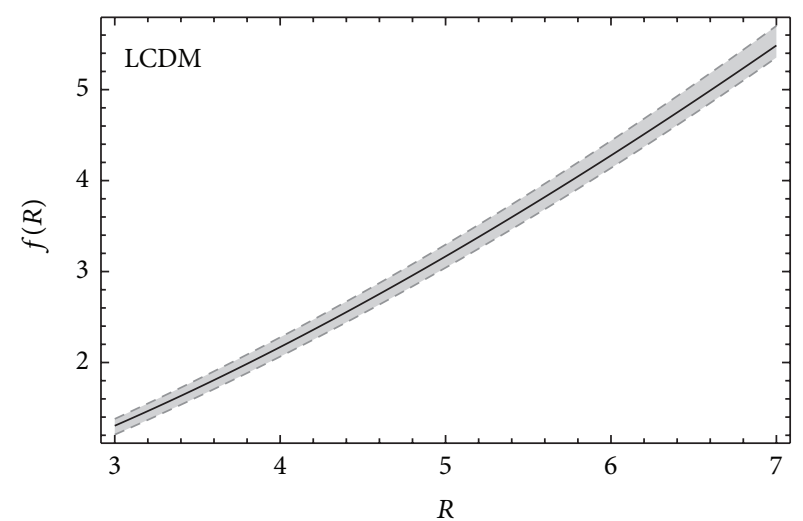

(b)

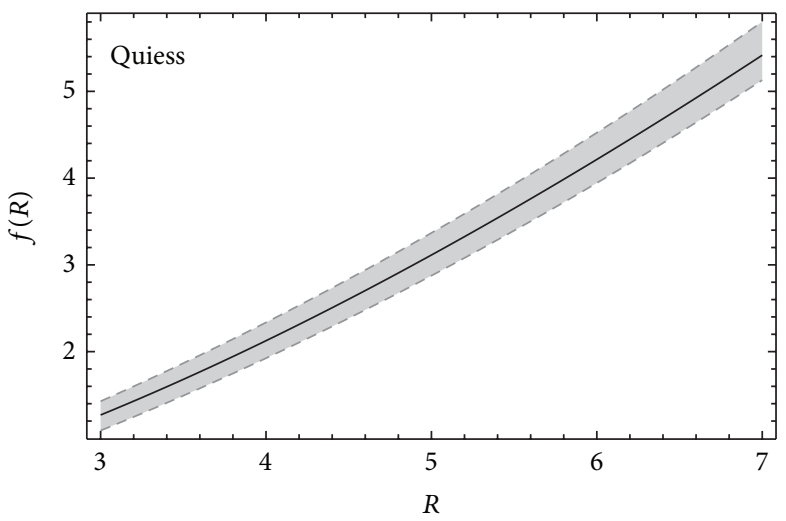

(c)

FIGURE 2: Gray band demonstrates permitted $f(R)$ for each model. The black line is analytically calculated $f(R)$, (15), in which we replaced $\alpha$ by that of Dark Energy.

TABLe 1: $H(z)^{2}$ for Dark Energy models [81].

\begin{tabular}{lrr}
\hline Model & $H(z)^{2}$ & Best fit parameter \\
\hline GCG & $H^{2}(z)=H_{0}^{2}\left[\Omega_{0 m}(1+z)^{3}+\left(1-\Omega_{0 m}\right)\left(a_{1}+\left(1-a_{1}\right)(1+z)^{3}\right)^{\omega}\right]$ & $a_{1}=0.9992_{-0.0060}^{+0.0008}$ \\
LCDM & $H^{2}(z)=H_{0}^{2}\left[\Omega_{0 m}(1+z)^{3}+1-\Omega_{0 m}\right]$ & $\omega=18.13 \pm 4.95$ \\
Quiess & $H^{2}(z)=H_{0}^{2}\left[\Omega_{0 m}(1+z)^{3}+\left(1-\Omega_{0 m}\right)(1+z)^{3(1+\omega)}\right]$ & $\Omega_{0 m}=0.31 \pm 0.04$ \\
& & $\omega=-1.02 \pm 0.11$ \\
\hline
\end{tabular}

From Figure 1 it is obvious that for $i>3$, the Taylor expansion coefficients have only slight effects.

\section{Reconstruction of $f(R)$ for Dark Energy Model}

In this section we have related Dark Energy models to cosmographic parameters by using Taylor expansion and then compared the result with the $f(R)$ function calculated in Section 3. For reconstructing $f(R)$ for Dark Energy models we need to use corresponding $H(z)^{2}$ s from Table 1, as follows:

(1) Using $H(z)^{2}$ we have calculated $q(z)$ for each Dark Energy model.

(2) Red shift must be fixed to zero.
(3) Using (28) to calculate $\alpha$ for each Dark Energy model, and replacing it in (23) and (26), we complete the Taylor expansion.

In this paper we have used GCG, LCDM, and Quiess models, since their present value of Ricci scalar is approximately the same $\left(R_{0} \approx 4.43\right)$. As errors in Dark Energy model parameters cannot be ignored, we would reach to a group of possible $f(R)$ function for each model, which has been demonstrated as a gray band in Figure 2. For comparing results, we have plotted analytically calculated action from (15), with these diagrams, where we have replaced $\alpha$ by that of Dark Energy according to the values of Table 2. Seeing that, for a linear correction equation (10), both analytically calculated $f(R)(15)$ and reconstructed $f(R)$ for Dark Energy models are in good agreement in neighboring with the present day value of curvature scalar. 
TABLE 2: Parameters for Dark Energy models with $H_{0}=0.697$.

\begin{tabular}{lcccc}
\hline & $q_{0}$ & $R_{0}$ & $\alpha$ & $f^{\prime \prime}\left(R_{0}\right)$ \\
\hline GCG & $-0.48528_{-0.06472}^{+0.10067}$ & $4.32025_{-0.28257}^{+0.19777}$ & $0.40817_{-0.04082}^{+0.06208}$ & $0.12294_{-0.00249}^{+0.00163}$ \\
LCDM & $-0.55000 \pm 0.06000$ & $4.51802 \pm 0.17489$ & $0.36636 \pm 0.04000$ & $0.12059 \pm 0.00171$ \\
Quiess & $-0.57100 \pm 0.11550$ & $4.57924 \pm 0.33666$ & $0.34950 \pm 0.07848$ & $0.120375 \pm 0.00313$ \\
\hline
\end{tabular}

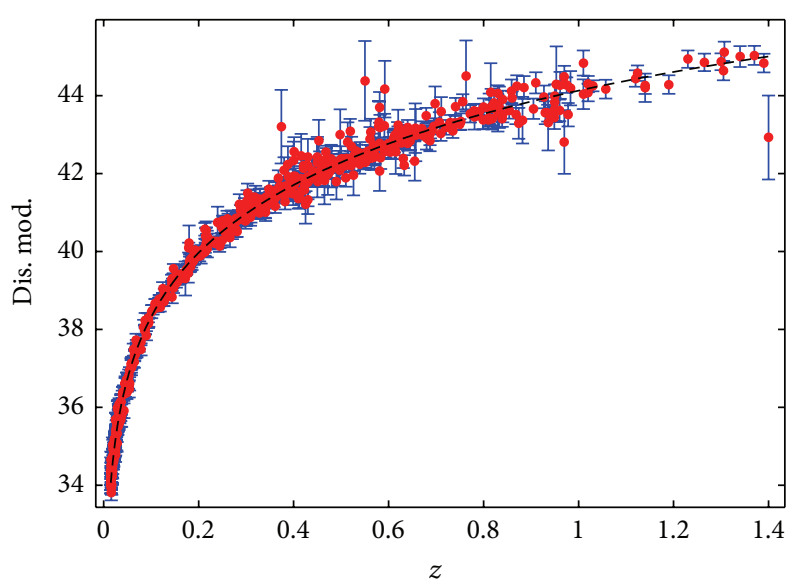

FIgURE 3: Accordance between Union2 SNeIa data and late time linear correction of our model for $\alpha \approx 0.418$ and $\mathrm{H}_{0} \approx 0.697$ [62].

\section{Conclusions}

Here we presented a model independent approach to calculate $f(R)$ by relating it to the present day value of cosmographic parameters. Since we had a linear form of $F(z)$, the $\eta_{\mathrm{i} 0} \mathrm{~s}$ values made us using Taylor expansion up to the third order. In higher order $F(z)$ corrections it might be possible to keep higher terms of Taylor expansion which depend on value of $\eta_{\mathrm{i} 0} \mathrm{~s}$ for these corrections and then we would discuss about validity of these terms in compare with desirable conditions. The Taylor expansion based $f(R)$ functions are viable in a small range about a certain value of Ricci scalar which itself is related to cosmographic parameters; since only present day value of these parameters are derived from data, we cannot discuss behavior of Universe at high red shifts.

\section{References}

[1] S. Perlmutter, G. Aldering, G. Goldhaber et al., "Measurements of $\Omega$ and $\Lambda$ from 42 high-redshift Supernovae," Astrophysical Journal Letters, vol. 517, no. 2, pp. 565-586, 1999.

[2] A. G. Riess, A. V. Filippenko, P. Challis et al., "Observational evidence from supernovae for an accelerating universe and a cosmological constant," Astronomical Journal, vol. 116, no. 3, pp. 1009-1038, 1998.

[3] S. Cole, W. J. Percival, J. A. Peacock et al., "The 2dF Galaxy Redshift Survey: power-spectrum analysis of the final data set and cosmological implications," Monthly Notices of the Royal Astronomical Society, vol. 362, no. 2, pp. 505-534, 2005.

[4] G. Hinshaw, M. R. Nolta, and C. L. Bennett, "Three-year Wilkinson Microwave Anisotropy Probe (WMAP) observations: temperature analysis," The Astrophysical Journal Supplement, vol. 170, p. 288, 2007.

[5] D. N. Spergel, R. Bean, O. Doré et al., "Wilkinson Microwave Anisotropy Probe (WMAP) three year results: implications for cosmology," The Astrophysical Journal, vol. 170, p. 377, 2007.

[6] U. Seljak, A. Makarov, P. McDonald et al., "Cosmological parameter analysis including SDSS Ly-alpha forest and galaxy bias: constraints on the primordial spectrum of fluctuations, neutrino mass, and dark energy," Physical Review, vol. 71, Article ID 103515, 20 pages, 2005.

[7] D. J. Eisenstein, I. Zehavi, D. W. Hogg et al., "Detection of the Baryon acoustic peak in the large-scale correlation function of SDSS luminous red galaxies," The Astrophysical Journal, vol. 633, p. 560, 2005.

[8] B. Jain and A. Taylor, "Cross-correlation tomography: measuring dark energy evolution with weak lensing," Physical Review Letters, vol. 91, Article ID 141302, 2003.

[9] E. J. Copeland, M. Sami, S. Tsujikawa et al., "Dynamics of dark energy," International Journal of Modern Physics D, vol. 15, p. 1753, 2006.

[10] M. Sami, "A primer on problems and prospects of dark energy," Current Science, vol. 97, p. 887, 2009.

[11] V. Sahni and A. Starobinsky, "The case for a positive cosmological $\Lambda$-term," International Journal of Modern Physics D, vol. 9, no. 4, pp. 373-443, 2000.

[12] T. Padmanabhan, "Cosmological constant-the weight of the vacuum," Physics Reports, vol. 380, pp. 235-320, 2003.

[13] E. V. Linder, "The dynamics of quintessence, the quintessence of dynamics," General Relativity and Gravitation, vol. 40, pp. 329356, 2008.

[14] J. Frieman, M. Turner, and D. Huterer, "Dark energy and the accelerating universe," Annual Review of Astronomy and Astrophysics, vol. 46, pp. 385-432, 2008.

[15] R. Caldwell and M. Kamionkowski, "The physics of cosmic acceleration," Annual Review of Nuclear and Particle Science, vol. 59, pp. 397-429, 2009.

[16] A. Silvestri and M. Trodden, "Approaches to understanding cosmic acceleration," Reports on Progress in Physics, vol. 72, Article ID 096901, 2009.

[17] C. Armendariz-Picon, T. Damour, and V. Mukhanov, " $k$-inflation," Physics Letters B, vol. 458, pp. 209-218, 1999.

[18] J. Garriga and V. F. Mukhanov, "Perturbations in $k$-inflation," Physics Letters Section B, vol. 458, no. 2-3, pp. 219-225, 1999.

[19] T. Chiba, T. Okabe, and M. Yamaguchi, "Kinetically driven quintessence," Physical Review D, vol. 62, Article ID 023511, 2000.

[20] C. Armendariz-Picon, V. Mukhnov, and P. J. Steinhardt, "Dynamical solution to the problem of a small cosmological constant and late-time cosmic acceleration," Physical Review Letters, vol. 85, pp. 4438-4441, 2000. 
[21] C. Armendariz-Picon, V. Mukhnov, and P. J. Steinhardt, "Essentials of k-essence," Physical Review D, vol. 63, Article ID 103510, 2001.

[22] T. Chiba, “Tracking k-essence," Physical Review D, vol. 66, Article ID 063514, 2002.

[23] L. P. Chimento and A. Feinstein, "Powert-low expansion in $k$ essence cosmology," Modern Physics Letters A, vol. 19, p. 761, 2004.

[24] L. P. Chimento, "Extended tachyon field, chaplygin gas and solvable $k$-essence cosmologies," Physical Review D, vol. 69, Article ID 123517, 2004.

[25] R. J. Scherrer, "Purely kinetic $k$ essence as unified dark matter," Physical Review Letters, vol. 93, Article ID 011301, 2004.

[26] A. Kamenshchik, U. Moschella, and V. Pasquier, "An alternative to quintessence," Physics Letters Section B, vol. 511, no. 2-4, pp. 265-268, 2001.

[27] N. Bilic, G. B. Tupper, and R. D. Viollier, "Unification of dark matter and dark energy: the inhomogeneous Chaplygin gas," Physics Letters B, vol. 535, pp. 17-21, 2002.

[28] M. C. Bento, O. Bertolami, and A. A. Sen, "Generalized Chaplygin gas, accelerated expansion, and dark-energy-matter unification," Physical Review D, vol. 66, Article ID 043507, 2002.

[29] A. Dev, J. S. Alcaniz, and D. Jain, "Cosmological consequences of a Chaplygin gas dark energy," Physical Review D, vol. 67, Article ID 023515, 2003.

[30] V. Gorini, A. Kamenshchik, and U. Moschella, "Can the Chaplygin gas be a plausible model for dark energy?" Physical Review D, vol. 67, no. 6, Article ID 063509, 2003.

[31] R. Bean and O. Dore, "Are Chaplygin gases serious contenders for the dark energy?" Physical Review D, vol. 68, Article ID 23515, 2003.

[32] T. Multamaki, M. Manera, and E. Gaztanaga, "Large scale structure and the generalised Chaplygin gas as dark energy," Physical Review D, vol. 69, Article ID 023004, 2004.

[33] A. A. Sen and R. J. Scherrer, "Generalizing the generalized Chaplygin gas," Physical Review D, vol. 72, Article ID 063511, 2005.

[34] G. Dvali, G. Gabadadze, and M. Porrati, “4D gravity on a brane in 5D Minkowski space," Physics Letters Section B, vol. 485, no. 1-3, pp. 208-214, 2000.

[35] G. R. Dvali, G. Gabadadze, M. Kolanovic, and F. Nitti, "The power of brane-induced gravity," Physical Review D, vol. 64, Article ID 084004, 2001.

[36] G. R. Dvali, G. Gabadadze, M. Kolanovic, and F. Nitti, "Scales of gravity," Physical Review D, vol. 64, Article ID 024031, 2002.

[37] A. Lue, R. Scoccimarro, and G. Starkman, "Differentiating between modified gravity and dark energy," Physical Review D, vol. 69, no. 4, Article ID 044005, 2004.

[38] A. Lue, R. Scoccimarro, and G. Starkman, "Probing Newton's constant on vast scales: DGP gravity, cosmic acceleration, and large scale structure," Physical Review D, vol. 69, Article ID 124015, 2004.

[39] P. Caresia, S. Matarrese, and L. Moscardini, "Constraints on extended quintessence from high-redshift supernovae," Astrophysical Journal, vol. 605, no. 1, pp. 21-28, 2004.

[40] V. Pettorino, C. Baccigalupi, and G. Mangano, "Extended quintessence with an exponential coupling," Journal of Cosmology and Astroparticle Physics, vol. 2002, article 014, 2005.

[41] M. Demianski, E. Piedipalumbo, C. Rubano, and C. Tortora, "Accelerating universe in scalar tensor models-comparison of theoretical predictions with observations," Astronomy and Astrophysics, vol. 454, no. 1, pp. 55-66, 2006.

[42] S. Thakur, A. A. Sen, and T. R. Seshadri, "Non-minimally coupled $f(R)$ cosmology," Physics Letters B, vol. 696, pp. 309314, 2011.

[43] S. Capozziello, "Curvature quintessence," International Journal of Modern Physics D, vol. 11, pp. 483-492, 2002.

[44] S. Capozziello, V. F. Cardone, S. Carloni, and A. Troisi, "Curvature quintessence matched with observational data," International Journal of Modern Physics D, vol. 12, no. 10, pp. 1969-1982, 2003.

[45] S. Capozziello, V. F. Cardone, and A. Trosi, "Reconciling dark energy models with $f(R)$ theories," Physical Review D, vol. 71, Article ID 043503, 2005.

[46] S. Carloni, P. K. S. Dunsby, S. Capozziello, and A. Troisi, "Cosmological dynamics of Rn gravity," Classical and Quantum Gravity, vol. 22, no. 22, pp. 4839-4868, 2005.

[47] H. Kleinert and H. J. Schmidt, "Cosmology with curvaturesaturated gravitational lagrangian $R / \sqrt{1+l^{4} R^{2}}$," General Relativity and Gravitation, vol. 34, pp. 1295-1318, 2002.

[48] S. Nojiri and S. D. Odintsov, "Where new gravitational physics comes fromml: M-theory?" Physics Letters B, vol. 576, p. 5, 2003.

[49] S. Nojiri and S. D. Odintsov, "The minimal curvature of the universe in modified gravity and conformal anomaly resolution of the instabilities," Modern Physics Letters A, vol. 19, pp. 627638, 2003.

[50] S. Nojiri and S. D. Odintsov, "Modified gravity with negative and positive powers of curvature: Unification of inflation and cosmic acceleration," Physical Review D, vol. 68, Article ID 12352, 2003.

[51] S. M. Carroll, V. Duvvuri, M. Trodden, and M. Turner, "Is cosmic speed-up due to new gravitational physics?" Physical Review D, vol. 70, Article ID 043528, 2004.

[52] G. Allemandi, A. Borowiec, and M. Francaviglia, "Accelerated cosmological models in Ricci squared gravity," Physical Review D, vol. 70, no. 10, Article ID 103503, p. 1, 2004.

[53] S. Nojiri and S. D. Odintsov, "Introduction to modified gravity and gravitational alternative for dark energy," International Journal of Geometric Methods in Modern Physics, vol. 4, p. 115, 2007.

[54] S. Capozziello and M. Francaviglia, "Extended theories of gravity and their cosmological and astrophysical applications," General Relativity and Gravitation, vol. 40, no. 2-3, pp. 357-420, 2008.

[55] V. Faraoni, " $f(R)$ theories of gravity," Reviews of Modern Physics, vol. 82, pp. 451-497, 2010.

[56] W. Hu and I. Sawicki, "Cosmological constraints on $f(R)$ acceleration models," Physical Review D, vol. 76, Article ID 064004, 2007.

[57] A. A. Starobinsky, "Disappearing cosmological constant in $f(R)$ gravity, JETP Letters, vol. 86, no. 3, pp. 157-163, 2007.

[58] S. A. Appleby and R. A. Battye, "Do consistent $f(R)$ models mimic general relativity plus Lambda?" Physics Letters B, vol. 654, pp. 7-12, 2007.

[59] S. Nojiri and S. D. Odintsov, "Newton law corrections and instabilities in $f(R)$ gravity with the effective cosmological constant epoch," Physics Letters B, vol. 652, pp. 343-348, 2007.

[60] S. Tsujikawa, "Observational signatures of $f(R)$ dark energy models that satisfy cosmological and local gravity constraints," Physical Review D, vol. 77, Article ID 023507, 2008. 
[61] R. Hashemi and R. Saffari, "A well-behaved $f(R)$ gravity model in planetary motions," Planetary and Space Science, vol. 59, no. 4, pp. 338-342, 2011.

[62] S. Asgari and R. Saffari, "A model of $f(R)$ gravity as an alternative for dark matter in spiral galaxies," Applied Physics Research, vol. 2, pp. 99-102, 2010.

[63] R. Saffari and S. Rahvar, "Consistency condition of spherically symmetric solutions in $f(R)$ gravity," Modern Physics Letters A, vol. 24, p. 305, 2009.

[64] R. Saffari and S. Rahvar, " $f(R)$ gravity: from the pioneer anomaly to cosmic acceleration," Physical Review D, vol. 77, Article ID 104028, 2008.

[65] S. Baghram and S. Rahvar, "Inverse problem: reconstruction of modified gravity action in Palatini formalism by Supernova Type Ia data," Physical Review D, vol. 80, Article ID 124049, 2009.

[66] S. Nojiri and S. D. Odintsov, "Modified $f(R)$ gravity consistent with realistic cosmology: from matter dominated epoch to dark energy universe," Physical Review D, vol. 74, Article ID 086005, 2006.

[67] S. Nojiri and S. Odintsov, "Unifying inflation with LambdaCDM epoch in modified $f(R)$ gravity consistent with Solar system tests," Physics Letters B, vol. 657, pp. 238-245, 2007.

[68] G. Cognola, E. Elizalde, S. Nojiri, S. D. Odintsov, and L. Sebastiani, "A class of viable modified $f(R)$ gravities describing inflation and the onset of accelerated expansion," Physical Review D, vol. 77, Article ID 046009, 2008.

[69] E. Elizade, S. Nojiri, S. D. Odintsov, L. Sebastiani, and L. Zerbini, "Nonsingular exponential gravity: a simple theory for earlyand late-time accelerated expansion," Physical Review D, vol. 83, Article ID 086006, 2011.

[70] S. Nojiri and S. D. Odintsov, "Modified gravity and its reconstruction from the universe expansion history," Journal of Physics, vol. 66, Article ID 012005, 2007.

[71] S. Nojiri, S. D. Odintsov, and D. Saez-Gomez, "Cosmological reconstruction of realistic modified $f(R)$ gravities," Physics Letters B, vol. 681, no. 1, pp. 74-80, 2009.

[72] S. Nojiri and S. D. Odintsov, "Unified cosmic history in modified gravity: from $f(R)$ theory to Lorentz non-invariant models," Physics Reports, vol. 505, pp. 59-144, 2011.

[73] S. Asgari and R. Saffari, "Vacuum solution of a linear red-shift based correction in $f(R)$ gravity," General Relativity and Gravitation, vol. 44, no. 3, pp. 737-750, 2012.

[74] Z. Girones, A. Marchetti, O. Mena, C. Pena-Garay, and N. Rius, "Cosmological data analysis of $f(R)$ gravity models," Journal of Cosmology and Astroparticle Physics, vol. 2010, article 004, 2010.

[75] S. Capozziello, V. F. Cardone, and V. Salzano, "Cosmography of $f(R)$ gravity," Physical Review D, vol. 78, Article ID 063504, 2008.

[76] S. Nojiri and S. D. Odintsov, "Modified gravity with negative and positive powers of curvature: Unification of inflation and cosmic acceleration," Physical Review D, vol. 68, Article ID 123512, 2003.

[77] S. Nojiri and S. D. Odintsov, "Modified gravity with $\ln \mathrm{R}$ terms and cosmic acceleration," General Relativity and Gravitation, vol. 36, no. 8, pp. 1765-1780, 2004.

[78] G. J. Olmo, "Post-Newtonian constraints on $f(R)$ cosmologies in Palatini formalism," Physical Review D, vol. 72, Article ID 083505, 2005.

[79] S. Baghram, M. Farhang, and S. Rahvar, "Modified gravity with $f(R)=\sqrt{R^{2}-R_{0}^{2}}$, Physical Review D, vol. 75, no. 4, Article ID 044024, 2007.
[80] M. S. Movahed, S. Baghram, and S. Rahvar, "Consistency of $f(R)=\sqrt{R^{2}-R_{0}^{4}}$ gravity with cosmological observations in the Palatini formalism," Physical Review D, vol. 76, Article ID 044008, 2007.

[81] R. Lazkoz, S. Nesseris, and L. Perivolaropoulos, "Exploring cosmological expansion parametrizations with the gold snIa dataset," Journal of Cosmology and Astroparticle Physics, vol. 2005, article 010, 2005.

[82] T.P. Sotirio and V. Faraoni, " $f(R)$ theories of gravity," Reviews of Modern Physics, vol. 82, pp. 451-497, 2010. 

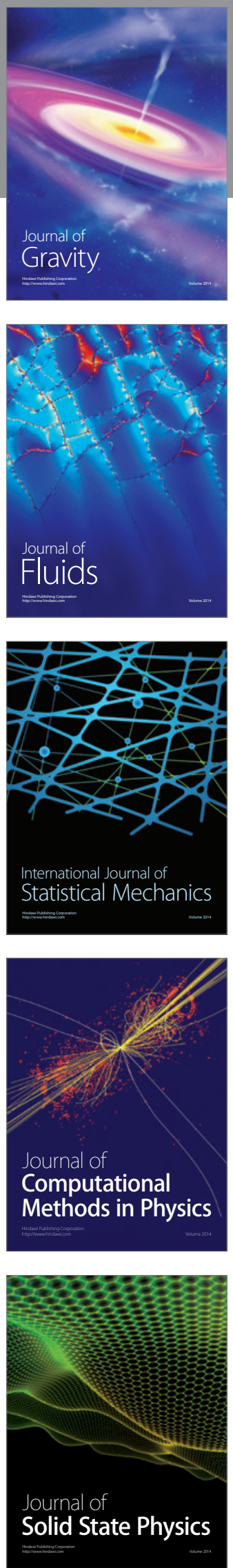

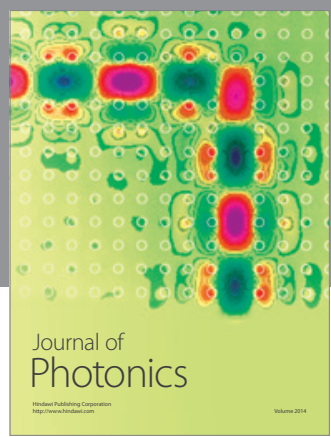

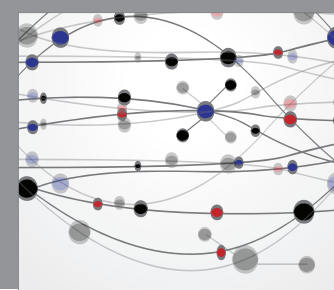

The Scientific World Journal

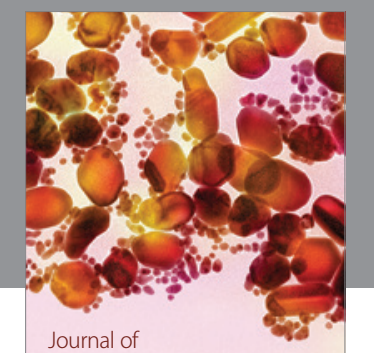

Soft Matter
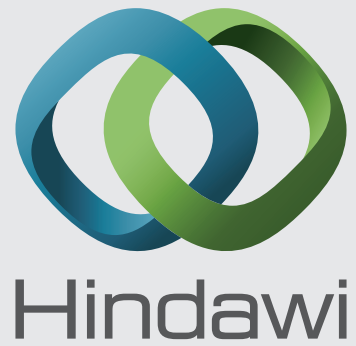

Submit your manuscripts at

http://www.hindawi.com
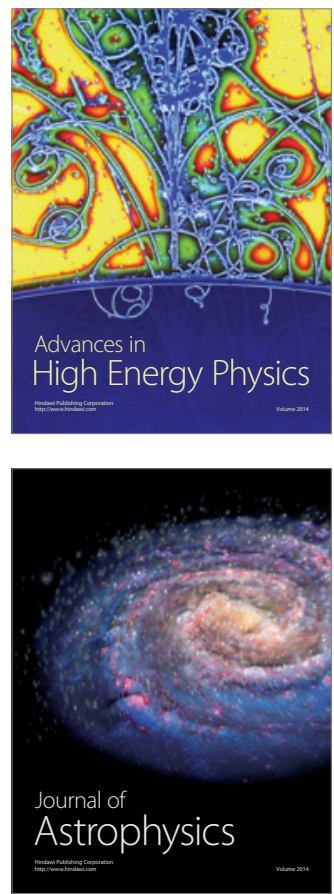
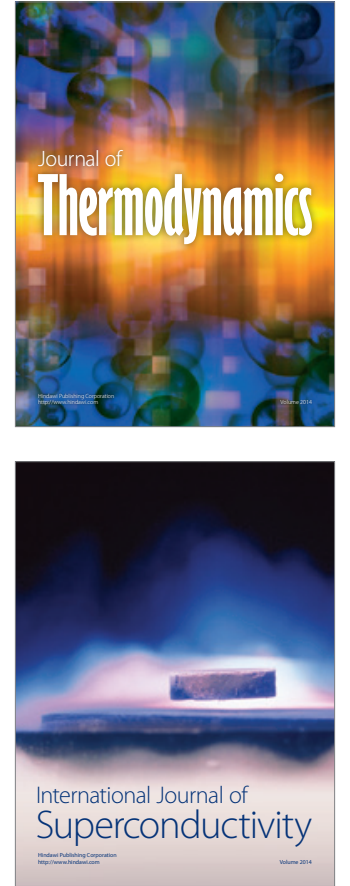
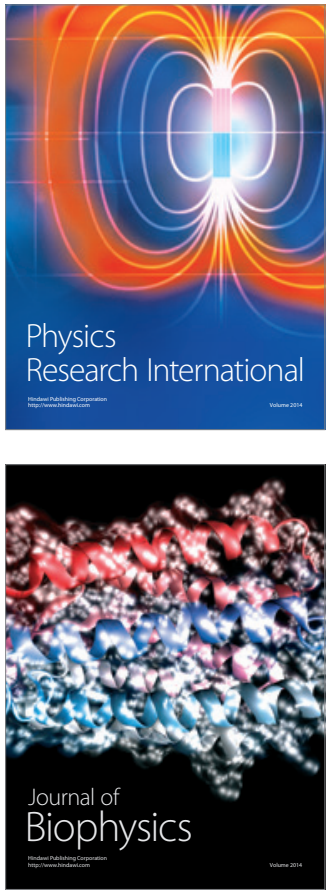
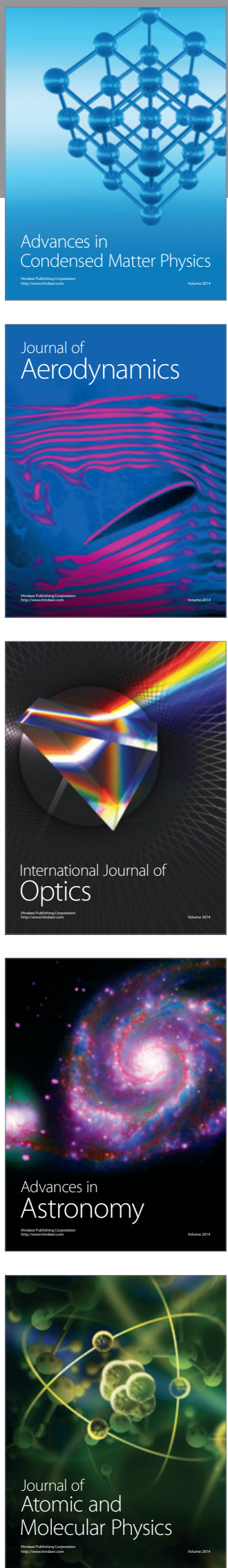